\section{Análisis estructural prospectivo: variables clave para el desarrollo organizacional de fundación de acción social Cáritas}

\section{Prospective structural analysis: key variables for the organiza- tional development of the Cáritas social action foundation}

\author{
Cristian Germán Hernández;' Joshua Hurtado Hurtado² \\ 1 Pontificia Universidad Católica del Ecuador, sede Santo Domingo \\ 2 Instituto de Investigación, Innovación y Estudios de Posgrado para la Educación del Estado (IIIEPE) de Nuevo \\ León, México
}

\section{Resumen}

El objetivo del presente estudio es desarrollar un análisis estructural prospectivo con el propósito de identificar las variables clave para el desarrollo organizacional de Fundación de Acción Social Cáritas, ubicada en la provincia de Santo Domingo de los Tsáchilas. Para este fin, se conformó un panel de expertos con el apoyo de coeficientes de competencia K. Una vez escogidos los 12 participantes, se desarrollaron diversos talleres de reflexión colectiva para crear un listado de variables de alta representación, validarlo y posteriormente procesarlo en el software especializado de la Escuela Francesa de Prospectiva denominado MICMAC (Matriz de Impactos Cruzados y Multiplicación Aplicada a una Clasificación). Como hallazgo principal, en el Plano de Influencias/dependencias Indirectas se encontraron 9 variables clave: alianzas estratégicas, capacidad de cobertura, capital tecnológico, complementariedad de los servicios, cuotas de recuperación, donaciones percibidas, estrategias de marketing social, formación del talento humano y sistema interno de comunicación. Por lo mencionado, se puede concluir que para el largo plazo la fundación requiere, en mayor medida, de una potenciación interna que puede abordarse de forma sistémica debido a la afinidad y complementariedad de las variables.

Palabras clave: Análisis estructural prospectivo; desarrollo organizacional; panel de expertos; MICMAC.

\section{Abstract}

The objective of this study is to develop a prospective structural analysis with the purpose of identifying the key variables for the organizational development of Fundación de Acción Social Cáritas, located in the province of Santo Domingo de los Tsáchilas. For this purpose, a panel of experts was formed with the support of coefficients of competence $\mathrm{K}$. Once the 12 participants had been chosen, various workshops for collective reflection were developed to create a list of highly represented variables, validate it and later process it in the software. specialized from the French School of Prospective called MICMAC (Matrix of Crossed Impacts and Multiplication Applied to a Classification). As a main finding, in the Plane of Indirect Influences / dependencies, 9 key variables were found: strategic alliances, coverage capacity, technological capital, complementary services, recovery fees, received donations, social marketing strategies, training of human talent and internal communication system. Therefore, it can be concluded that for the long term the foundation requires, to a greater extent, an internal empowerment that can be addressed in a systemic way due to the affinity and complementarity of the variables.

Keywords: Prospective structural analysis; organizational development; Expert Panel; MICMAC. 


\section{INTRODUCCIÓN}

Las Organizaciones No Gubernamentales son entidades de iniciativa social y fines humanitarios que no se rigen a una visión utilitarista o economicista para el desarrollo de sus operaciones. Por ello, de acuerdo con Baker (2002), se podrían caracterizar como actores centrales en una sociedad global y, según Hochstetler y Friedman (2008), en América Latina pueden representar un mecanismo de influencia sobre el entorno político a los ciudadanos. En cuanto a su forma jurídica, Gavilanes (2017), señala que en Ecuador estas estructuras están presentes como asociaciones, fundaciones, corporaciones, entre otras. Sin embargo, esto no es un factor que condicione su misión de brindar asistencia igualitaria y oportuna para promover el desarrollo socio-económico de los más vulnerables.

De acuerdo a Sánchez y Villarroel (2017), la importancia y la visibilidad de las ONG se deben a su vinculación con la sociedad civil y el conjunto de valores comunes que traslucen en los procesos de transformación social impulsados por estas en diversas épocas históricas. En Ecuador, el siglo XX fue el centro neurálgico para la creación de organizaciones enfocadas en la promoción de derechos y la equiparación de oportunidades y capacidades. No obstante, en la actualidad siguen representando una notable base de apoyo para los ciudadanos que viven en condiciones de pobreza o han visto vulnerado alguno de sus derechos. Esto resuena con la postura de Nikkhah y Redzuan (2010), quienes señalan que las ONG contribuyen al desarrollo comunitario sostenible, con Edwards (1999) considerando que estas instituciones pueden tener éxito en hacer una diferencia sobre las condiciones de vida de las personas en situación de pobreza si promueven formas de organización local y las vinculan con los mercados y con estructuras políticas que operan en un nivel superior.

Para Fowler (1993), las ONG necesitan 1) mantener una identidad propia, con misión y valores autónomos, 2) poseer una capacidad para lograr la satisfacción de las partes interesadas, y 3) administrar las interacciones externas para ser efectivas. Lograr lo anterior es retador, debido a los intereses opuestos de diferentes actores en el contexto social. Para su financiamiento, dependen de sus relaciones con agentes externos y donantes, lo que deriva, en palabras de Rauh (2010), en asimetrías que generan un comportamiento de pasividad, aunque algunas tienen la capacidad de negociar y resistir estas asimetrías. En particular, Reith (2010) sostiene que el poder en las relaciones donantes-ONG se ejerce mediante el control del flujo de dinero, y la práctica de recaudación de fondos perpetúa este desequilibrio de poder. No obstante, Burchell y Cook (2013) indican que diferentes formas de diálogo con las partes interesadas permiten una transformación de las relaciones de las ONG con sus donantes. De igual manera, Shumate y O'Connor (2010), señalan que la comunicación entre ONG y las empresas donantes no sólo fortalece la capacidad de operación de las ONG, sino que también puede movilizar capital cultural, económico, político y social entre la red de partes interesadas. Estas consideraciones sugieren que hay dinámicas de administración estratégica y comunicación que las ONG pueden adoptar para fortalecer su capacidad de agencia.

En cuanto a la provincia de Santo Domingo de los Tsáchilas, su tamaño poblacional comprende un total de 456244 habitantes, de los cuales, según cifras del Gobierno Autónomo Descentralizado Provincial de Santo Domingo de los Tsáchilas (2015), el $30,14 \%$ se encasilla en la categoría de pobreza por ingresos y el 74,36\% por necesidades básicas insatisfechas. Esta escasez de recursos deriva en otros inconvenientes como la falta de acceso a atención médica oportuna y de calidad, elevados niveles de delincuencia y un considerable ausentismo escolar. En vista de que las iniciativas gubernamentales no han sido suficientes para mitigar estos inconvenientes, las ONG contribuyen poniendo a disposición de la ciudadanía sus programas de asistencia acorde a la capacidad de cobertura y al tipo de servicio disponible.

Por lo mencionado, el objetivo de la presente investigación es desarrollar un análisis estructural prospectivo con el propósito de identificar las variables clave para el desarrollo organizacional de Fundación de Acción Social Cáritas. Se considera a esta ONG como foco de estudio debido a que es la de mayor relevancia en la provincia 
de acuerdo a su capacidad de cobertura, cartera de servicios diversificada y número de programas sociales vigentes. En conjunto, estos se enfocan en la preservación y restitución de vínculos familiares, la protección de los derechos de salud, nutrición y recreación, el fortalecimiento e impulso de la producción y comercialización agrícola, el mejoramiento de capacidades y calidad de vida de las personas y el impulso a emprendimientos familiares a través de microcréditos.

El llevar a cabo estudios prospectivos es particularmente valioso como un componente de la administración estratégica de las ONG, puesto que, de acuerdo con Vecchiato (2012), permiten a los gerentes detectar oportunidades y riesgos a tiempo y reaccionar ante estos estímulos de manera efectiva. Para von der Gracht y otros (2010), la prospectiva también facilita a las organizaciones compararse con sus pares e identificar áreas de oportunidad para desarrollo e innovación organizacional. Dicho esto, los estudios prospectivos genéricos pueden tener una aplicación limitada debido a la ausencia de vínculos claros entre los resultados de la prospectiva y la toma de decisiones. Por ello, según van der Steen y van Twist (2012), los consultores prospectivistas deben asesorar cuidadosamente a la organización receptora del estudio con respecto a los resultados y sus vínculos con la toma de decisiones, así como contextualizar los resultados para ofrecer mayor valor. En este sentido, la presente investigación buscó apoyar a la ONG receptora a navegar en el presente contexto de incertidumbre en términos económicos y de salud pública.

La relevancia del estudio radica en tres motivos principales. Primero, comprende a una organización con un número de beneficiarios relevante. Por citar un ejemplo, FASCA brindó sus servicios a aproximadamente 7000 personas adicionales en 2019. Segundo, se prevé que las donaciones provenientes de empresas privadas y cooperación internacional disminuirán para los próximos años a causa de la emergencia sanitaria por COVID-19 y, a su vez, por la reestructuración de la normativa para las ONG que, según Chiriboga (2014), empezó a enfatizar en el control de operaciones institucionales y transferencias internacionales a partir de la aprobación de la Constitución de la República en 2008. Y, como tercer motivo, en el país no existe registro de un estudio para una ONG que esté fundamentado en la Escuela Francesa de Prospectiva y su enfoque voluntarista. Por lo tanto, la construcción metodológica permitirá reconocer los puntos neurálgicos que deberá potenciar FASCA para mantener su normal funcionamiento post pandemia. Además, la propuesta favorecerá su replicación en otros contextos a causa de su rigurosidad y precisa concatenación entre fases.

\section{METODOLOGÍA}

$\mathrm{Al}$ tratarse de un análisis estructural prospectivo, la presente investigación se enmarca en el enfoque mixto. En términos de Cruz y Medina (2015), este es uno de los métodos cuali-cuantitativos de alcance descriptivo de mayor uso en la Escuela Francesa de Prospectiva y se caracteriza por su potencial para categorizar las variables representativas de un sistema según sus niveles de influencia y dependencia. Además, como señalan Pimienta y De la Orden (2017), corresponde a un diseño no experimental, en vista de que se analizan los fenómenos en su contexto natural y sin generar alteraciones deliberadas sobre las variables de estudio. En adición, Gándara y Osorio (2017), resaltan la indispensable aplicación del método MICMAC (Matriz de Impactos Cruzados y Multiplicación Aplicada a una Clasificación) para incursionar por las diversas fases del estudio con la guía que brindan los ejercicios de reflexión colectiva desarrollados junto a un panel de expertos.

\section{Formación del panel de expertos}

De acuerdo a Zartha, Halal y Hernández (2019), los métodos prospectivos requieren de dos tipos de agentes para su desarrollo: investigadores prospectivistas y expertos en el ámbito. Con respecto a estos últimos, su aporte consiste en la emisión de opiniones fundamentadas para incrementar la comprensión sistémica de una organización. Por lo tanto, como sugieren Matheus, Romero y Parroquín (2018), 
se empleó un instrumento para determinar los coeficientes de competencia K a 15 expertos potenciales. A su vez, con este instrumento se identificaron los coeficientes de conocimiento y argumentación de los expertos potenciales. Los primeros, se midieron a través de una escala ascendente (1 mínimo y 10 máximo), conforme a los niveles de dominio del tema de estudio. Mientras que, para los segundos, la relevancia recayó sobre los análisis teóricos realizados por los expertos, la experiencia obtenida en su actividad profesional, los estudios de autores nacionales y extranjeros que han revisado, el conocimiento propio acerca del estado del problema a abordar y su intuición sobre el tema.

Manteniendo como guía al estudio de Cruz y Martínez (2020), se trabajó con los siguientes rangos de clasificación: coeficiente alto $(0,8<\mathrm{K}<1.0)$, coeficiente medio $(0.5$ $<\mathrm{K}<0.8)$ y coeficiente bajo $(\mathrm{K}<0.5)$. Por consiguiente, para conformar el panel de expertos que contribuyó en esta investigación, fueron considerados solo aquellos que obtuvieron un coeficiente de competencia $\mathrm{K}$ alto, dando paso a la estructuración de una coalición con académicos, empresarios, servidores públicos y colaboradores de Fundación de Acción Social Cáritas. Así, como indican para Meijering y Tobi (2016), se conformó un panel multidisciplinario, favoreciendo al desenvolvimiento de una reflexión participativa con altos niveles de aprendizaje.

\section{Compresión del sistema}

Los estudios que involucran un análisis estructural prospectivo se fundamentan en el enfoque de la teoría de sistemas. Como explican Domínguez y López (2017), esto consiste en delimitar con precisión el sistema a ser intervenido para descomponerlo en variables con alta representación. En este caso, al tratarse de una organización, se siguen los lineamientos de Mojica (2005) y se desarrolla un diagnóstico situacional, abarcando así un análisis interno y externo con el apoyo de herramientas de gestión estratégica. De igual forma, junto al panel de expertos se llevó a cabo un ejercicio para la construcción de un árbol de competencias. Finalmente, se conformó colectivamente un listado inicial de 27 variables que en un proceso posterior fue validado.

\section{Validación de variables}

En este punto, al contar con una cantidad considerable de variables, fue primordial validarlas para ingresar solo elementos neurálgicos del sistema en el software de prospectiva. De esta forma, según Hernández y Cisneros (2020), se evita la duplicación de variables y se descarta de manera consensuada a aquellas con excesiva autonomía. Por lo tanto, se indagó en la literatura especializada, por ejemplo: Godet (1993), Popper (2011), Medina, Becerra y Castaño (2014) y Ortega (2016), acerca del método más adecuado, considerando la cantidad de expertos y la extensión del listado de variables inicial. De ahí que se haya optado por el Ábaco de Regnier, especialmente por su no exigencia de una cantidad mínima de 30 expertos, tal como requieren otros métodos de consulta grupal.

Él Abaco de Regnier se empleó con su escala cromática tradicional (Figura 1). En este nuevo taller, se entregó a los expertos un formato para depurar el listado de variables inicial. Es decir, según su experiencia e intuición, seleccionaron las variables de mayor importancia y descartaron aquellas que podían ser abarcadas por una de mayor representatividad. De este modo, con base en Bezold (2010), se procedió a ordenar los resultados obtenidos de forma descendente y se mantuvieron las variables en las que predominaron las votaciones con colores entre verde y amarillo; en contraste, la asignación de colores rosa y rojo equivalieron a un descarte directo de 5 variables.

\section{Validación de variables}

En cuanto a la última fase, fue imprescindible el uso del software MICMAC versión 6.1.2, desarrollado por LIPSOR (Laboratoire d'Investigation en Prospective, Stratégie et Organisation), 3IE (Institut d'Innovation Informatique pour l'entreprise) y EPITA (Ecole pour l'Informatique et les Techniques Avancées). Para Hernández (2020), este método representa el primer escalón dentro del proceso prospectivo y provee el insumo 


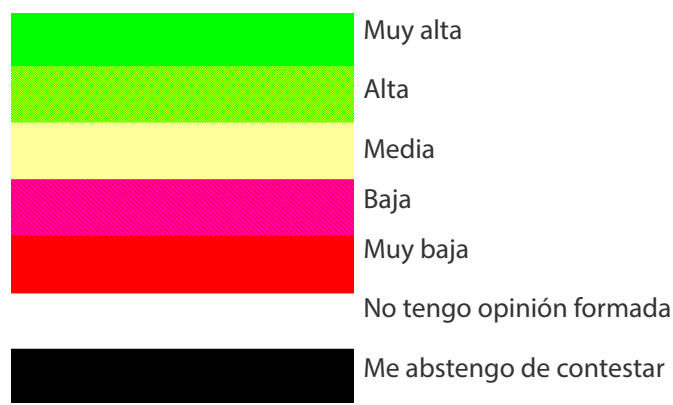

Figura 1. Escala cromática del Ábaco de Regnier Fuente: Ortega (2016)

de mayor valor para las fases subsecuentes: el Plano de Influencias/dependencias Indirectas. De acuerdo a Ariyani y Fauzi (2019), el correcto desarrollo de la Matriz de Influencias Directas es esencial para obtener hallazgos precisos, puesto que las ponderaciones asignadas deben ser debatidas y analizadas por el conjunto de expertos acorde a los niveles de influencia de una variable sobre otra. Para este fin, Akbar, Azadi, Dehghani, Lebailly y Qafori, (2019) facilitan las siguientes calificaciones: $0=$ no influye, 1 = influencia débil, 2 = influencia moderada, 3 = influencia fuerte y $\mathrm{P}=$ influencia potencial. Por último, se recurrió al plano guía para categorizar variables que proporcionan Godet y Durance (2011) (Figura 2).

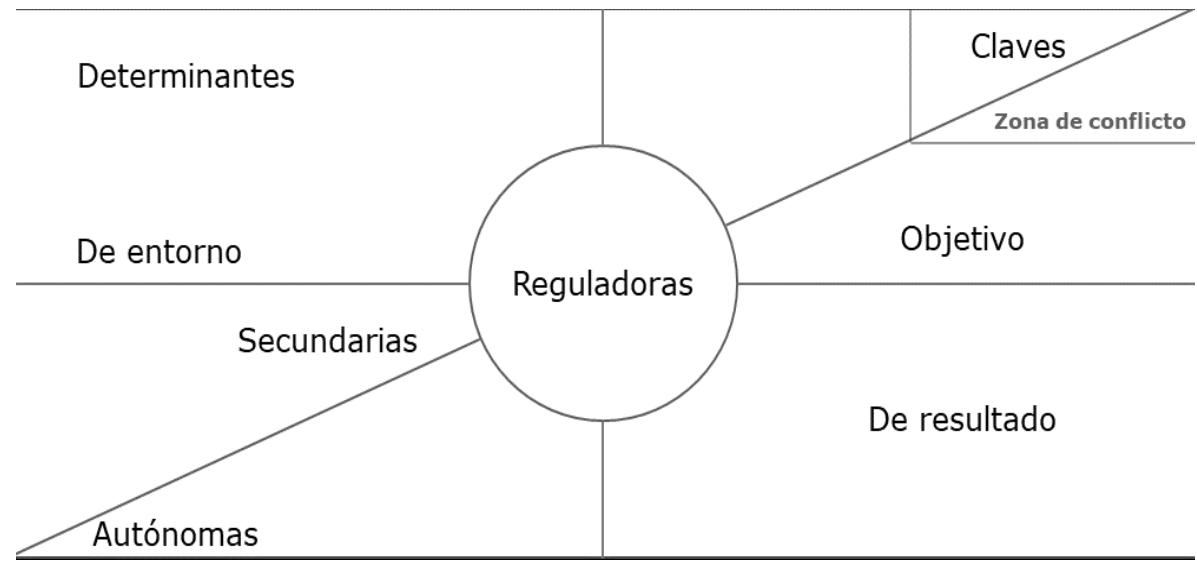

Figura 2. Plano guía para categorizar variables en MICMAC Fuente: Godet y Durance (2011).

\section{RESULTADOS}

Los hallazgos obtenidos durante el desarrollo procedimental inician con la conformación del panel de expertos. Así, con la aplicación de un instrumento enfocado en los coeficientes de competencia $\mathrm{K}$ fue posible clasificar a los expertos potenciales. Como resultado relevante, tres de los quince participantes obtuvieron una calificación media; es decir, entre 0,5 y 0,8. Por otra parte, los doce restantes presentaron puntuaciones superiores a 0,85 y fueron invitados a formar parte de la coalición (Tabla 1). Por consiguiente, el panel de expertos cuenta con coeficiente promedio igual a 0,89 , lo cual permite aseverar que los ejercicios de reflexión colectiva se desarrollarán bajo el consenso de un grupo multidisciplinario y con opinión formada y especializada en el ámbito. 
Tabla 1. Selección de los expertos

\begin{tabular}{ccccc}
\hline $\begin{array}{c}\text { Experto } \\
\text { potencial }\end{array}$ & $\begin{array}{c}\text { Coeficiente de } \\
\text { conocimiento } \\
(\mathbf{K c})\end{array}$ & $\begin{array}{c}\text { Coeficiente de } \\
\text { argumentación } \\
\text { (Ka) }\end{array}$ & $\begin{array}{c}\text { Coeficiente de } \\
\text { competencia } \\
\text { (K) }\end{array}$ & Calificación \\
\hline 1 & 0.7 & 0.8 & 0.75 & Medio \\
2 & 1 & 0.9 & 0.95 & Alto \\
3 & 0.8 & 0.9 & 0.85 & Alto \\
4 & 0.8 & 0.9 & 0.85 & Alto \\
5 & 0.9 & 0.9 & 0.9 & Alto \\
6 & 0.6 & 0.7 & 0.65 & Medio \\
7 & 0.8 & 1 & 0.9 & Alto \\
8 & 0.8 & 0.9 & 0.85 & Alto \\
9 & 1 & 0.9 & 0.95 & Alto \\
10 & 0.5 & 0.7 & 0.6 & Medio \\
11 & 0.8 & 0.9 & 0.85 & Alto \\
12 & 0.9 & 0.9 & 0.9 & Alto \\
13 & 0.9 & 0.9 & 0.9 & Alto \\
14 & 0.9 & 0.8 & 0.85 & Alto \\
15 & 0.8 & 1 & 0.9 & Alto \\
\hline
\end{tabular}

Nota. La fórmula para calcular los coeficientes de competencia $(\mathrm{K})$ corresponde a $\mathrm{K}=1 / 2(\mathrm{Kc}+\mathrm{Ka})$.

En cuanto a la comprensión del sistema, fue de gran apoyo el desarrollo de un diagnóstico situacional de la fundación, en vista de que esta únicamente había trabajado en función de planes operativos anuales. De esta forma, con la integración de diversas herramientas para el análisis interno y externo, se procedió a descomponer a la organización en un conjunto de variables representativas. En este primer taller participativo, los expertos identificaron un total de 27 variables como parte de un listado tentativo. Posteriormente, en un nuevo taller, se aplicó el instrumento de validación denominado Ábaco de Regnier. Para la resolución de este, los expertos emplearon la escala de colores tradicional del instrumento y, con la premisa de evitar la duplicación de variables y eliminar aquellas con baja representación, se descartaron cuatro variables que reflejaban colores rojos y ningún verde acentuado (Tabla 2).

Como resultado del proceso anterior, se obtuvo el listado definitivo de variables con un total de veintidós (Tabla 3). En este punto, sobresale la necesidad de recursos percibidos mediante donaciones, puesto que corresponde a la fuente principal de ingresos para Fundación de Acción Social Cáritas. De igual forma, los expertos han enfatizado en la examinación de cuatro perspectivas clásicas en gestión estratégica: financiera, del cliente, del proceso interno y de formación y crecimiento. Sumado a esto, el estudio de la estructura orgánica, el ciclo de vida de los servicios, la cadena de valor y el mapa de procesos (parte interna), junto con un análisis PESTEL y el desarrollo de las fuerzas competitivas de Porter (parte externa), han brindado el soporte necesario para condensar apropiadamente a la organización.

Con respecto a la Matriz de Influencias Directas (Tabla 4), fue necesario llevarla a cabo en dos talleres participativos debido a su amplitud. Para esto, mediante un proceso de causalidad reflexiva que busca el consenso de los participantes, se realizaron un total de 462 ponderaciones al evaluar los niveles de incidencia de cada una de las variables en relación a las demás. Como se observa, los expertos no han considerado ninguna influencia potencial y han plasmado relaciones que van desde influencia nula (0) hasta influencia fuerte (3). Esto se debe a que las variables que condicionan las posibilidades de desarrollo de la organización son conocidas y mantienen influencias bidireccionales establecidas. 
Tabla 2. Aplicación del Ábaco de Regnier

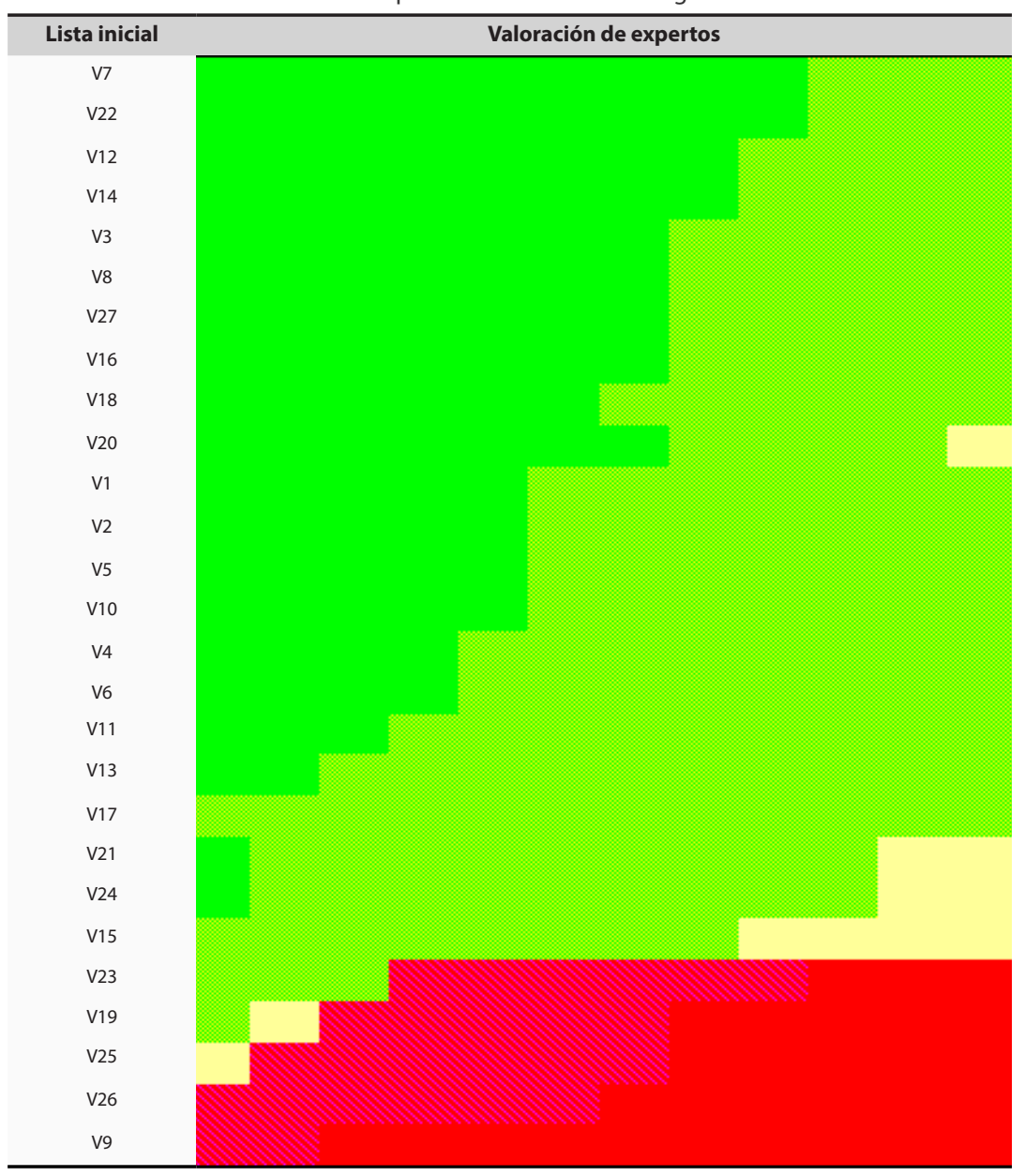

Tabla 3. Lista validada de variables

\begin{tabular}{|c|c|c|}
\hline No. & Variables & Clave \\
\hline 1 & Actividades primarias & AP \\
\hline 2 & Alianzas estratégicas & $\mathrm{AE}$ \\
\hline 3 & Aplicación de herramientas de gestión estratégica & AHGE \\
\hline 4 & Asesoría internacional & $\mathrm{Al}$ \\
\hline 5 & Capacidad de cobertura & $\mathrm{CC}$ \\
\hline 6 & Capital tecnológico & $\mathrm{CT}$ \\
\hline 7 & Certificaciones & CE \\
\hline 8 & Ciclo de vida de los servicios & CVS \\
\hline 9 & Complementariedad de los servicios & CS \\
\hline 10 & Cultura organizacional & $\mathrm{CO}$ \\
\hline 11 & Cuotas de recuperación & $\mathrm{CR}$ \\
\hline 12 & Donaciones percibidas & DP \\
\hline 13 & Estrategias de marketing social & EMS \\
\hline 14 & Estructura orgánica & EO \\
\hline 15 & Formación del talento humano & FTH \\
\hline 16 & Necesidades futuras de la comunidad & NFC \\
\hline 17 & Poder de negociación con proveedores & PNP \\
\hline 18 & Procesos estratégicos & PE \\
\hline 19 & Programas de voluntariado & PV \\
\hline 20 & Regulaciones político-legales & RPL \\
\hline 21 & Representación jurídica & RJ \\
\hline 22 & Sistema interno de comunicación & SIC \\
\hline
\end{tabular}


Tabla 4. Matriz de Influencias Directas

\begin{tabular}{|c|c|c|c|c|c|c|c|c|c|c|c|c|c|c|c|c|c|c|c|c|c|c|}
\hline & AP & AE & AHGE & $\mathrm{Al}$ & CC & CT & CE & CVS & CS & $\mathrm{CO}$ & CR & DP & EMS & EO & FTH & NFC & PNP & $\mathrm{PE}$ & PV & RPL & $\mathrm{RJ}$ & SIC \\
\hline AP & 0 & 2 & 1 & 1 & 2 & 3 & 1 & 1 & 2 & 1 & 2 & 2 & 2 & 1 & 3 & 1 & 1 & 1 & 1 & 1 & 1 & 2 \\
\hline $\mathrm{AE}$ & 2 & 0 & 2 & 1 & 2 & 2 & 2 & 2 & 2 & 3 & 3 & 2 & 3 & 2 & 3 & 3 & 3 & 2 & 3 & 2 & 2 & 2 \\
\hline AHGE & 1 & 3 & 0 & 1 & 2 & 3 & 1 & 1 & 3 & 1 & 3 & 3 & 3 & 1 & 3 & 1 & 1 & 1 & 1 & 1 & 1 & 3 \\
\hline $\mathrm{Al}$ & 1 & 1 & 1 & 0 & 2 & 2 & 0 & 0 & 2 & 1 & 1 & 1 & 1 & 0 & 2 & 1 & 0 & 1 & 0 & 1 & 0 & 1 \\
\hline CC & 2 & 2 & 3 & 1 & 0 & 3 & 2 & 2 & 2 & 3 & 2 & 2 & 3 & 3 & 2 & 3 & 2 & 2 & 2 & 3 & 2 & 3 \\
\hline CT & 2 & 3 & 3 & 1 & 2 & 0 & 2 & 2 & 2 & 3 & 2 & 2 & 3 & 3 & 2 & 2 & 2 & 3 & 3 & 2 & 2 & 3 \\
\hline $\mathrm{CE}$ & 1 & 2 & 1 & 0 & 2 & 2 & 0 & 0 & 2 & 1 & 2 & 2 & 2 & 0 & 2 & 1 & 1 & 0 & 0 & 0 & 0 & 2 \\
\hline CVS & 1 & 2 & 1 & 0 & 2 & 2 & 0 & 0 & 2 & 1 & 2 & 2 & 2 & 0 & 2 & 1 & 0 & 0 & 0 & 1 & 0 & 2 \\
\hline CS & 2 & 3 & 3 & 1 & 2 & 2 & 2 & 2 & 0 & 3 & 3 & 2 & 3 & 3 & 2 & 3 & 2 & 2 & 2 & 2 & 2 & 2 \\
\hline $\mathrm{CO}$ & 1 & 2 & 1 & 1 & 3 & 2 & 1 & 1 & 2 & 0 & 3 & 3 & 2 & 1 & 3 & 1 & 1 & 1 & 1 & 1 & 1 & 3 \\
\hline$C R$ & 1 & 2 & 3 & 2 & 3 & 3 & 2 & 2 & 2 & 2 & 0 & 3 & 2 & 2 & 2 & 3 & 3 & 3 & 2 & 3 & 3 & 3 \\
\hline DP & 2 & 3 & 3 & 2 & 2 & 3 & 2 & 2 & 2 & 2 & 3 & 0 & 3 & 2 & 2 & 3 & 3 & 2 & 2 & 2 & 2 & 3 \\
\hline EMS & 2 & 3 & 3 & 2 & 2 & 2 & 2 & 2 & 3 & 3 & 2 & 3 & 0 & 3 & 3 & 3 & 3 & 2 & 2 & 3 & 3 & 2 \\
\hline EO & 1 & 2 & 1 & 0 & 2 & 2 & 0 & 0 & 3 & 1 & 3 & 3 & 3 & 0 & 3 & 1 & 0 & 0 & 0 & 0 & 0 & 2 \\
\hline FTH & 2 & 3 & 3 & 1 & 3 & 3 & 2 & 2 & 2 & 2 & 2 & 3 & 3 & 3 & 0 & 2 & 2 & 3 & 2 & 3 & 2 & 3 \\
\hline NFC & 1 & 3 & 1 & 1 & 3 & 3 & 1 & 1 & 3 & 1 & 2 & 2 & 3 & 0 & 2 & 0 & 1 & 1 & 1 & 1 & 1 & 3 \\
\hline PNP & 1 & 2 & 1 & 1 & 3 & 3 & 1 & 1 & 2 & 1 & 3 & 2 & 2 & 1 & 3 & 1 & 0 & 1 & 1 & 1 & 1 & 3 \\
\hline PE & 1 & 2 & 1 & 1 & 3 & 3 & 1 & 1 & 2 & 1 & 2 & 2 & 2 & 1 & 3 & 1 & 1 & 0 & 1 & 1 & 1 & 3 \\
\hline PV & 1 & 3 & 1 & 1 & 2 & 2 & 1 & 1 & 3 & 1 & 2 & 2 & 2 & 1 & 3 & 1 & 1 & 1 & 0 & 1 & 1 & 3 \\
\hline RPL & 1 & 3 & 1 & 1 & 3 & 3 & 1 & 1 & 3 & 1 & 2 & 3 & 3 & 1 & 2 & 1 & 1 & 1 & 1 & 0 & 1 & 2 \\
\hline RJ & 1 & 1 & 1 & 0 & 1 & 1 & 0 & 0 & 1 & 1 & 1 & 1 & 1 & 0 & 1 & 1 & 1 & 0 & 1 & 0 & 0 & 1 \\
\hline SIC & 2 & 3 & 3 & 1 & 3 & 2 & 2 & 2 & 3 & 2 & 2 & 2 & 3 & 2 & 3 & 2 & 3 & 2 & 3 & 2 & 3 & 0 \\
\hline
\end{tabular}

El hallazgo neurálgico recae sobre el Plano de Influencias/dependencias Indirectas (Figura 3), debido a que brinda una categorización de variables con el apoyo de subdivisiones que facilitan su interpretación. Por consiguiente, la evidencia refleja que las variables clave para el desarrollo organizacional de Fundación de Acción Social Cáritas corresponden a las alianzas estratégicas, la capacidad de cobertura, el capital tecnológico, la complementariedad de los servicios, las cuotas de recuperación, las donaciones percibidas, las estrategias de marketing social, la formación del talento humano y el sistema interno de comunicación. Según lo observado, estas variables se encuentran en la zona de conflicto del sistema, lo cual significa que son capaces de perturbar el normal funcionamiento de la organización e incluso pueden complicar su existencia en el largo plazo. Por lo tanto, al ser críticas, deben ser consideradas como los retos a futuro para alcanzar la condición ideal de la fundación.

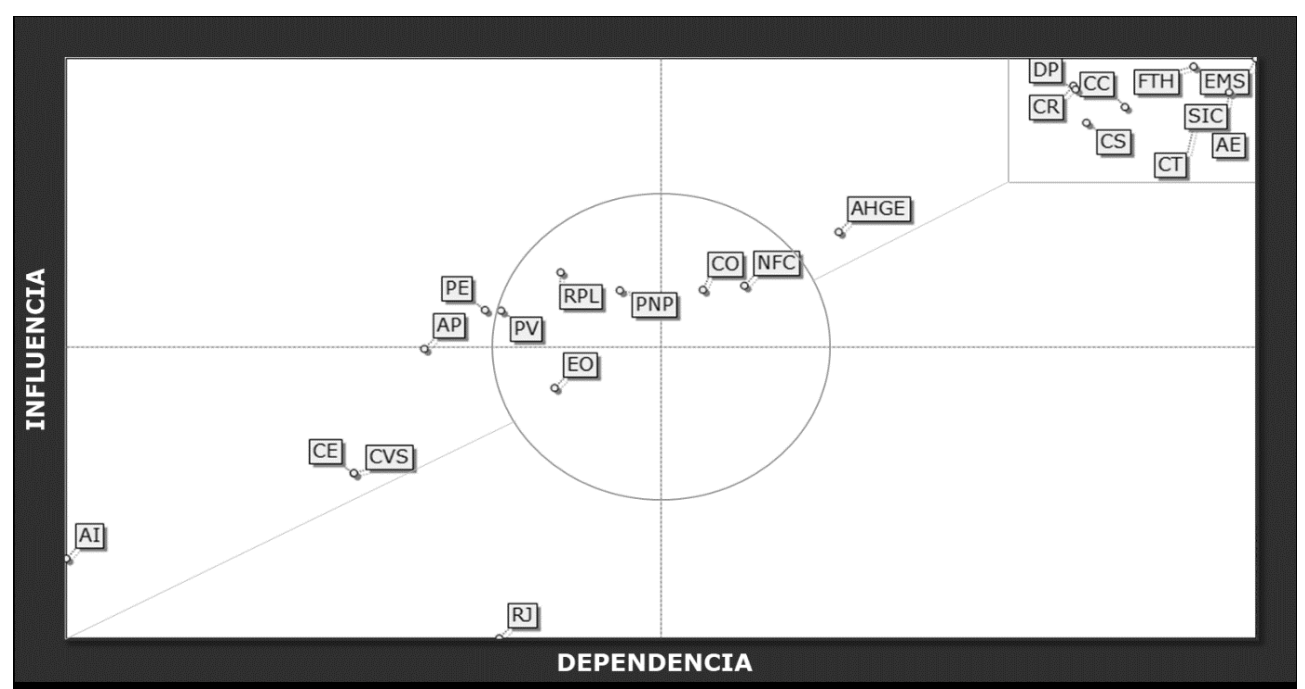

Figura 3. Plano de Influencias/dependencias Indirectas resultante 
En lo concerniente a las demás categorizaciones, se encontró que las variables reguladoras corresponden a cultura organizacional, estructura orgánica, necesidades futuras de la comunidad, poder de negociación con proveedores, programas de voluntariado y regulaciones político-legales. Por otra parte, las variables secundarias engloban a certificaciones, actividades primarias, ciclo de vida de servicios y representaciones jurídicas. Además, se encuentra solo a aplicación de herramientas de gestión estratégica como variable objetivo, procesos estratégicos como variable de entorno y asesoría internacional como variable autónoma. Finalmente, no se encontraron variables determinantes ni de resultado.

\section{DISCUSIONES}

Los resultados de las variables claves identificadas coinciden con hallazgos de investigaciones previas, aunque también reflejan un énfasis diferente en el caso de la Fundación de Acción Social Cáritas en el contexto ecuatoriano . Las alianzas estratégicas son vínculos indispensables para esta $\mathrm{ONG}$, al igual que lo son para las ONG que operan en otros ámbitos de competencia y contextos nacionales. Se debe recordar lo señalado por Edwards (1999) en lo respectivo al proceso que pueden seguir las ONG dedicadas al combate a la pobreza, dado que por medio de alianzas estratégicas se podrían generar vínculos de los beneficiarios de las ONG con los mercados y con estructuras políticas que les ayuden a salir de la condición de pobreza. Lo anterior coincide también la investigación de Fowler (1993), quien argumenta que las ONG deben satisfacer a las diferentes partes interesadas y administrar sus interacciones externas, así como con la de Sánchez y Villarroel (2017), quienes enfatizan el papel de la vinculación de las ONG con la sociedad civil. La relevancia de las alianzas estratégicas también resuena con la perspectiva de Yanacopulos (2005) sobre la creación de una coalición de ONG, la cual puede ser bastante influyente políticamente e incidir en el entorno deseado.

La capacidad de cobertura está íntimamente relacionada con las alianzas estratégicas, por lo cual su aparición como variable clave es lógica. De acuerdo con Edwards y Hulme (1992), quienes facilitaron un taller de estrategias de ONG enfocadas en el desarrollo, el trabajar con entidades del gobierno, hacer cabildeo en las esferas de toma de decisiones, y buscar el surgimiento y fortalecimiento de organizaciones locales pueden incrementar el impacto de la ONG mediante beneficios generados de manera indirecta. Para formar alianzas estratégicas y aumentar la capacidad de cobertura se requiere de capital tecnológico, algo que fortalece tanto las operaciones como la formación de coaliciones. Esto encuentra sustento en los estudios de Bach y Stark (2002; 2004), quienes proponen que las tecnologías interactivas basadas en internet pueden proporcionar un medio de representación en la esfera pública e incrementar la innovación organizacional. Advierten, sin embargo, que no se debe suponer un uso instrumental de la tecnología por parte de las ONG; en cambio, sugieren que las ONG y las tecnologías co-evolucionan según el contexto.

Desde el punto de vista de operaciones y sostenibilidad financiera, se pueden explicar la aparición de las variables complementariedad de los servicios, cuotas de recuperación y donaciones percibidas. Para Iglesias y Carreras (2013), la complementariedad de los servicios es importante para aprovechar el talento del personal, reducir los gastos de operación, y focalizar las acciones de la ONG para maximizar el impacto. La complementariedad, asimismo, deberá incorporar la visualización de las acciones de otros actores institucionales en el entorno para fortalecer la acción propia de la ONG. A su vez, las cuotas de recuperación se posicionan como variable clave debido a que permiten cierto grado de autonomía de las entidades donantes y sostienen las operaciones de la ONG. Esto concuerda con la postura de Alymkulova y Seipulnik (2005), quienes indican que las cuotas de recuperación por servicios contribuyen a la sostenibilidad financiera de las ONG. Las cuotas de recuperación son de particular importancia ya que, como indicaron Rauh (2010) y Reith (2010), las ONG están en una posición dependiente de las empresas donantes. 
Ante este panorama, también sería importante considerar la diversificación de donantes y fuentes de ingresos, algo también propuesto por Alymkulova y Seipulnik (2005). No obstante, como argumenta Mitchell (2014), se deben combinar diferentes estrategias para fortalecer la sostenibilidad financiera y operaciones de las ONG, tales como: comercialización, arbitraje geoestratégico, especialización de la ONG, selectividad con respecto a los beneficiarios, educación de los donantes, y la negociación estratégica.

Por estas razones es que las tres variables claves restantes - estrategias de marketing social, formación del talento humano, y el sistema interno de comunicación - son igualmente medulares en una visión a futuro, porque las tres contribuyen a mejorar el desempeño de las operaciones de la ONG y a la comunicación con las partes interesadas, tanto aliados estratégicos como beneficiarios. Tanto Burchell y Cook (2013) como Schumate y O'Connor (2010) resaltan el papel de las estrategias de comunicación externa, pues pueden reducir las asimetrías entre donantes y ONG, así como movilizar diferentes formas de capital, lo que permite potenciar el impacto de las actividades de las ONG. Para maximizar el impacto de las ONG, una fuerza laboral profesional también es necesaria, pues de acuerdo con Hilton y otros (2013), contribuyen a direccionar la pericia hacia sitios de incidencia política. Finalmente, según Fitzpatrick (2018), un sistema interno de comunicación efectivo promueve un mejor desempeño en el ámbito organizacional, debido a que aseguran que los miembros de la ONG comprendan y se adhieran a los protocolos y compartan ideas en torno a la innovación de procesos y servicios.

\section{CONCLUSIONES}

Este estudio prospectivo ha tenido como intención fortalecer el desarrollo organizacional de Fundación de Acción Social Cáritas, para lo cual ha sido crucial identificar las variables clave sobre las cuales esta ONG tendrá que incidir. El primer hallazgo relevante es que todas las variables clave en la zona de conflicto del sistema (alianzas estratégicas, la capacidad de cobertura, el capital tecnológico, la complementariedad de los servicios, las cuotas de recuperación, las donaciones percibidas, las estrategias de marketing social, la formación del talento humano y el sistema interno de comunicación) están vinculadas estratégicamente: son variables que se refuerzan entre sí y que están dentro del margen de acción de la Fundación de Acción Social Cáritas. Por ejemplo, las alianzas estratégicas y el capital tecnológico pueden ampliar la capacidad de cobertura de la ONG. Esto quiere decir que, en lugar de pensar en estas variables de manera individual, Fundación de Acción Social Cáritas deberá abordarlas desde una perspectiva sistémica.

Un segundo hallazgo es que la variable asesoría internacional (AI) se encuentra en el cuadrante de variables autónomas, lo que implica que no impacta ni gobierna a las demás variables. Esto es relevante porque la asesoría internacional generalmente es algo valioso para las ONG. En el caso de Fundación de Acción Social Cáritas, sin embargo, parece que lo prioritario y aquello en donde debe enfocar sus recursos es en las variables que consoliden su operación como ONG. Una vez que esté su desempeño evidencie cumplir con todas las metas propuestas por la administración se podría considerar ir más allá de este estudio y pensar en la asesoría internacional.

Finalmente, y relacionado con los dos hallazgos anteriores, se debe resaltar la contribución de los estudios de futuros a la toma de decisiones, particularmente en este caso. Debido a recursos limitados, una ONG como Fundación de Acción Social Cáritas debe de decidir hacia qué propósitos destinarlos. En este caso, la toma de decisión debe orientarse hacia aquellas variables que fortalezcan las operaciones, aunque en principio parezcan tener poca relación, tales como capital tecnológico, marketing social y el sistema interno de comunicación, entre otras. Aunque no parezcan estar vinculadas directamente con el impacto de la ONG objeto de este estudio, destinar recursos hacia ellas puede robustecer el desempeño de la ONG y, por ende, maximizar su impacto. 


\section{REFERENCIAS}

Akbar, A., Azadi, H., Dehghani, M., Lebailly, P., \& Qafori, M. (2019). Determining Key Agricultural Strategic Factors Using AHP-MICMAC. Sustainability(11), 2-17.

Alymkulova, A., \& Seipulnik, D. (2005). NGO Strategy for Survival in Central Asia: Financial Sustainability (No. 22). Michigan: The William Davidson Institute at the University of Michigan Business School.

Ariyani, N., \& Fauzi, A. (2019). Analysis of Strategic Variables for Ecotourism Development; an Application of MICMAC. South Asian Journal of Social Studies and Economics, 3(3), 2-12.

Bach, J., \& Stark, D. (2002). Innovative ambiguities: NGO's use of interactive technology in Eastern Europe. Studies in Comparative International Development, 37(2), 3-23. https://doi.org/10.1007/BF02686259

Bach, J., \& Stark, D. (2004). Link, Search, Interact: The Co-Evolution of NGOs and Interactive Technology. Theory, Culture \& Society, 21(3), 101-117. https://doi.org/10.1177/0263276404043622

Baker, G. (2002). Problems in the theorisation of global civil society. Political Studies, 50(5), 928-943. https://doi. org/10.1111/1467-9248.00401

Bezold, C. (2010). Lessons from using scenarios for strategic Foresight. Technological Forecasting and Social Change, $77(8), 1513-1518$.

Burchell, J., \& Cook, J. (2013). Sleeping with the Enemy? Strategic Transformations in Business - NGO Relationships Through Stakeholder Dialogue. Journal of Business Ethics, (113), 505-518. https://doi.org/10.1007/s10551-0121319-1

Chiriboga, M. (2014). Las ONG ecuatorianas en los procesos de cambio. Quito: Abya-Yala.

Cruz, M., \& Martínez, M. (2020). Origen y desarrollo de un índice de competencia experta: el coeficiente k. Revista Latinoamericana de Metodología de la Investigación Social, 10(19), 40-56.

Cruz, P., \& Medina, J. (2015). Selección de los métodos para la construcción de los escenarios de futuro. Entramado, 11(1), 32-46.

Domínguez, V., \& López, M. (2017). Teoría General de Sistemas, un enfoque práctico. Tecnociencia, 10(3), $125-132$.

Edwards, M., \& Hulme, D. (1992). Scaling up NGO impact on development: Learning from experience. Development in Practice, 2(2), 77-91. https://doi.org/10.1080/096145249100076691

Edwards, M., \& Bank, T. W. (1999). NGO Performance - What Breeds Success? New Evidence from South Asia. World Development, $27(2), 361-364$.

Fitzpatrick, L. (2018). Internal Communication in NGOs. In N. Garsten \& I. Bruce (Eds.), Communicating Causes: Strategic public relations for the non-profit sector. Abingdon: Routledge.

Fowler, A. (1993). Assessing NGO Performance: Difficulties, Dilemmas and a Way Ahead. In D. Hulme \& M. Edwards (Eds.), Beyond the Magic Bullet: NGO Performance and Accountability in the Post-Cold War World (pp. 143-156). Boulder, Colorado: Kumerian Press.

Gándara, G., \& Osorio, F. (2017). Métodos prospectivos: Manual para el estudio y la construcción del futuro. México: Paidós.

Gavilanes, M. (2017). Estado y ONG en Ecuador durante el período 2007-2014. Universidad Andina Simón Bolívar, Ecuador.

Gobierno Autónomo Descentralizado Provincial Santo Domingo de los Tsáchilas. (2015). Plan de Desarrollo y Ordenamiento Territorial Santo Domingo de los Tsáchilas 2015 - 2030.

Godet, M. (1993). De la anticipación a la acción: manual de prospectiva estratégica. Barcelona: Marcombo.

Godet, M., \& Durance, P. (2011). La prospectiva estratégica para las empresas y los territorios. París: DUNOD.

Iglesias, M., \& Carreras, I. (2013). La colaboración efectiva en las ONG : alianzas estratégicas y redes. ESADE / PWC.

Hernández Ordoñez, C. G., \& Cisneros Corrales, E. P. (2020). Estudio prospectivo: escenarios para Santo Domingo como territorio sostenible al año 2040. Tsafiqui Revista Científica en Ciencias Sociales, 11(14), 37-54. doi:10.29019/tsafiqui.v14i1.672

Hernández, C. (2020). Planificación estratégica prospectiva: métodos MACTOR y SMIC. Dimensión Empresarial, 18(1), 170-175. doi:10.15665/dem.v18i(1).2127

Hilton, M., McKay, J., Crowson, N., \& Mouhot, J.-F. (2013). The Politics of Expertise. How NGOs Shaped Modern Britain. Oxford: Oxford University Press.

Hochstetler, K., \& Friedman, E. J. (2008). Can civil society organizations solve the crisis of partisan representation in Latin America? Latin American Politics and Society, 50(2), 1-32. https://doi.org/10.1111/j.1548-2456.2008.00011.x

Matheus, A., Romero, R., \& Parroquín, P. (2018). Validación por expertos de un instrumento para la identificación de habilidades y competencias de un profesional en el área de logística. Cultura Científica y Tecnológica, 14(63), 227-238.

Medina, J., Becerra, S., \& Castaño, P. (2014). Prospectiva y política pública para el cambio estructural en América Latina y el Caribe. Santiago de Chile: CEPAL.

Meijering, J., \& Tobi, H. (2016). The effect of controlled opinion feedback on Delphi features: Mixed messages from a real-world Delphi experiment. Technologial Forecasting \& Social Change (103), 166-173.

Mitchell, G. E. (2014). Strategic Responses to Resource Dependence Among Transnational NGOs Registered in the United States. Voluntas, 25(1), 67-91. https://doi.org/10.1007/s11266-012-9329-2

Mojica, F. (2005). La construcción del futuro: concepto y modelo de prospectiva estratégica, territorial y tecnológica. Bogotá: Universidad Externado de Colombia.

Nikkhah, H. A., \& Redzuan, M. Bin. (2010). The Role of NGOs in Promoting Empowerment for Sustainable Community Development. Journal of Human Ecology, 30(2), 85-92. https://doi.org/10.1080/09709274.2010.119062 76

Ortega, F. (2016). Prospectiva empresarial: Manual de corporate foresight para América Latina. Lima: Universidad de Lima.

Pimienta, J. H., \& De la Orden, A. (2017). Metodología de la Investigación. México, D.F.: Pearson Educación.

Popper, R. (2011). Metodología de la Prospectiva. Manual de prospectiva tecnológica. Conceptos y prácticas. México: Facultad Latinoamericana de Ciencias Sociales. 
Rauh, K. (2010). NGOs, Foreign Donors, and Organizational Processes : Passive NGO Recipients or Strategic Actors? McGill Sociological Review, 1(January), 29-45.

Reith, S. (2010). Money, power, and donor-NGO partnerships. Development in Practice, 20(3), 446-455. https:// doi.org/10.1080/09614521003709932

Sánchez, M., \& Villaroel, R. (2017). Tensiones en la Intervención Social: (des)encuentros en la relación Estado-ONG. Estudio de caso sobre ONG que opera la política social de infancia. Revista Trabajo Social, 91, $3-16$.

Shumate, M., \& O’Connor, A. (2010). The symbiotic sustainability model: Conceptualizing NGO-corporate alliance communication. Journal of Communication, 60(3), 577-609. https://doi.org/10.1111/j.14602466.2010.01498.x

Van der Steen, M., \& Van Twist, M. (2012). Beyond use: Evaluating foresight that fits. Futures, 44(5), 475-486. https://doi.org/10.1016/j.futures.2012.03.009

Vecchiato, R. (2012). Environmental uncertainty, foresight and strategic decision making: An integrated study. Technological Forecasting and Social Change, 79(3), 436-447. https://doi.org/10.1016/j.techfore.2011.07.010

von der Gracht, H. A., Vennemann, C. R., \& Darkow, I. L. (2010). Corporate foresight and innovation management: A portfolio-approach in evaluating organizational development. Futures, 42(4), 380-393. https://doi. org/10.1016/j.futures.2009.11.023

Yanacopulos, H. (2005). The strategies that bind: NGO coalitions and their influence. Global Networks, 5(1), 93-110.

Zartha, J., Halal, W., \& Hernández, R. (2019). Delphi method: analysis of rounds, stakeholder and statistical indicators. Foresight, 21(5), 525-544. 